\title{
Survival and healthcare utilization of infants diagnosed with lethal congenital malformations
}

\author{
Jacqueline E. Nguyen ${ }^{1,2} \cdot$ Jason L. Salemi $\mathbb{1}^{3,4} \cdot$ Jean P. Tanner ${ }^{4} \cdot$ Russell S. Kirby $\mathbb{1}^{4} \cdot$ Ronald P. Sutsko ${ }^{1,5} \cdot$ \\ Terri L. Ashmeade ${ }^{1} \cdot$ Hamisu M. Salihu ${ }^{3,4} \cdot$ Laura L. Drach $^{6}$
}

Received: 2 May 2018 / Revised: 25 August 2018 / Accepted: 30 August 2018 / Published online: 20 September 2018

(c) Springer Nature America, Inc. 2018

\begin{abstract}
Objective: We assessed survival, hospital length of stay (LOS), and costs of medical care for infants with lethal congenital malformations, and also examined the relationship between medical and surgical therapies and survival.

Study design: Retrospective cohort study including infants born 1998-2009 with lethal congenital malformations, identified using a longitudinally linked maternal/infant database.

Results: The cohort included 786 infants: trisomy 18 (T18, $n=350)$, trisomy 13 (T13, $n=206)$, anencephaly $(n=125)$, bilateral renal agenesis $(n=53)$, thanatophoric dysplasia/achondrogenesis/lethal osteogenesis imperfecta $(n=38)$, and infants $>1$ of the birth defects $(n=14)$. Compared to infants without birth defects, infants with T18, T13, bilateral renal agenesis, and skeletal dysplasias had longer survival rates, higher inpatient medical costs, and longer LOS.

Conclusion: Care practices and survival have changed over time for infants with T18, T13, bilateral renal agenesis, and skeletal dysplasias. This information will be useful for clinicians in counseling families and in shaping goals of care prenatally and postnatally.
\end{abstract}

\section{Introduction}

The diagnosis of an infant with a congenital malformation conventionally described as "lethal" brings with it difficult

Electronic supplementary material The online version of this article (https://doi.org/10.1038/s41372-018-0227-3) contains supplementary material, which is available to authorized users.

Jacqueline E. Nguyen

jnguye31@jhmi.edu

1 Division of Neonatology, Morsani College of Medicine University of South Florida, Tampa, FL, USA

2 Division of Neonatology, Johns Hopkins All Children's Hospital, St. Petersburg, FL, USA

3 Department of Family and Community Medicine, Baylor College of Medicine, Houston, TX, USA

4 Department of Community and Family Health, College of Public Health, University of South Florida, Tampa, FL, USA

5 Division of Neonatology, Levine Children's Hospital/Atrium Health, Charlotte, NC, USA

6 Division of Hospice and Palliative Care, Johns Hopkins All Children's Hospital, St. Petersburg, FL, USA continuation vs. termination decisions for parents [1]. In addition, medical providers struggle with their own emotions and discomfort with uncertainty. Current practice by providers includes counseling parents that these diagnoses are either not compatible with survival beyond the newborn period, or for early survivors, are associated with extremely poor quality of a still-shortened life. Recent data describe longer survival rates for infants with lethal malformations than previously reported [1-4]. Wilkinson et al. [2]. presented survival data for infants with trisomy 18 (T18), trisomy 13 (T13), anencephaly, bilateral renal agenesis, and thanatophoric dysplasia using recent population cohort studies and case reports. Cases of survival to at least 6 months of age have been described for all of these conditions. Also, families of survivors of infants with T13 or T18 report positive family experiences, regardless of their child's length of survival [1, 3-5]. These studies, in addition to rapid technological advances, have led to a more active approach of offering intensive treatment vs. the traditional palliative/hospice care, non-interventional paradigm in some neonatal intensive care units. Studies of infants with T13 or T18 have shown that this trend towards more intensive management has led to increased survival rates $[4,6-14]$. 
This shift towards more intensive management has increased the ethical, legal, and financial challenges associated with the care of these infants, including the potential burden on healthcare resources and the possibility that these actions will only prolong suffering without providing significant benefit. There is a critical gap in knowledge regarding health services utilization, long-term survival, treatment interventions, and discharge status among infants with "lethal'" congenital malformations, particularly for infants with less-studied defects such as anencephaly, bilateral renal agenesis, and lethal skeletal dysplasias. Therefore, the purpose of this study was to assess length of survival and describe healthcare utilization practices by evaluating hospital length of stay (LOS) and institutional costs of direct medical care during the first year of life for infants with selected malformations often described as "lethal." We also examined associations between medical and surgical therapies and survival.

\section{Methods}

\section{Study design and population}

We conducted a retrospective cohort study leveraging data from a statewide, longitudinally linked maternal and infant database created using federal data infrastructure funds for comparative effectiveness research [15, 16]. Briefly, for each resident live birth in Florida from 1 January, 1998 through 31 December, 2009, we linked birth certificates to all maternal and infant hospital discharge data and to death certificates from birth through 31 December, 2010. Details of the hierarchical deterministic linking strategy, which achieved higher than a $92 \%$ linkage rate, and an evaluation of the validity and reliability of database have been published previously [17].

For this study, the initial study population consisted of all singleton infants born alive to Florida-resident mothers who were captured in the statewide linked database. We then restricted to infants who were delivered at 20-44 weeks gestation and excluded those with extreme birth weight ( < 125 or $>6000 \mathrm{~g}$ ) or implausible birth weight-for-gestational age, based on national fetal growth curves [18]. Then, using a combination of International Classification of Diseases, Ninth Edition, Clinical Modification (ICD-9-CM) and Tenth Edition (ICD-10) diagnosis and procedure codes, we classified infants into mutually exclusive groups based on the presence of one or more lethal congenital malformations: (1) trisomy 18, (2) trisomy 13, (3) anencephaly, (4) renal agenesis, (5) skeletal dysplasias, (6) $>1$ lethal malformation. Infants without these lethal malformations constituted the reference group. To meet the case definition, the diagnosis must have been made on a hospital discharge record within the first 30 days of life or as an underlying or contributing cause of death on the infant death certificate. Due to the lack of sufficient specificity of some ICD codes and in the absence of diagnostic verification through medical record review, the case definition for renal agenesis required an indication of oligohydramnios, and for skeletal dysplasias required an indication of pulmonary hypoplasia in addition to either respiratory failure or receipt of ventilation support. Specific codes used for each condition or procedure are described in Supplementary Table 1.

\section{Clinical outcomes}

We compared the frequency and rates of various medical diagnoses and clinical interventions for each defect group. Clinical outcomes included respiratory, cardiovascular, gastrointestinal, renal, infectious, orofacial, and neurological diseases with long-term morbidity, non-surgical interventions (e.g., invasive mechanical ventilation, non-invasive mechanical ventilation, long-term oxygen therapy, parenteral nutrition, intubation, chest tube or central line placement, peritoneal dialysis, IV antibiotics, blood transfusions, or palliative care), and surgical interventions (e.g., cardiac surgery, extracorporeal membrane oxygenation (ECMO), feeding tube, gastrointestinal surgeries, ventricular shunt placement, tracheostomies, and orofacial cleft repair). All clinical outcomes were identified using ICD-9CM diagnosis and procedure codes present in inpatient hospitalizations taking place during the first year of life (Supplementary Table 2).

An infant death was considered to have occurred if the infant either had (1) a linked death certificate in which the documented date of death was less than 365 days after the infant's date of birth, or (2) a linked hospital discharge record with a patient disposition documented as "expired" and in which the documented date of discharge (date of death) was less than 365 days after the date of birth. Survival time was then calculated as the number of days from date of birth to the date of death for infants who died during the first year of life, and as 365 for infants who survived the entire first year of life.

\section{Hospital utilization}

Among defect groups, we also explored two indicators of healthcare utilization: (1) the number of inpatient days in the hospital (i.e., LOS), and (2) cost of providing direct inpatient medical care. For both indicators, we assessed utilization during the birth hospitalization and during the entire first year of life. To calculate LOS in days, we first subtracted the dates of admission and discharge; same day visits with a calculated LOS of 0 were assigned an LOS of 1 day since same day hospitalizations (including inpatient) 
constitute a distinct stay and are also billed as 1 day for room/board [19]. Then, for each infant, the number of days across all admissions within the time frame of interest was summed. The linked database also contains departmentlevel (e.g., operating room) charges for each inpatient hospitalization. However, charges reflect only what a hospital bills for services and inaccurately estimates the actual cost of service provision [20]. The degree of markup from cost to billed charges varies considerably across hospitals, departments within the same hospital, and over time. Therefore, we used a previously published algorithm to convert charges to more reliable cost estimates using time-, hospital-, and department-specific cost-to-charge ratios (CCRs) from hospital cost reports, and we further adjusted estimates for inflation to 2010 dollars using the medical care component of the Consumer Price Index [20, 21].

\section{Covariates}

Maternal and infant sociodemographic and clinical characteristics for infants with and without birth defects were captured using birth certificates and discharge data. These characteristics included maternal age at delivery (in 5-year age groups), race/ethnicity, nativity (US vs. foreign-born), marital status, education, income, insurance status, pregnancy history, timing of prenatal care, method of delivery, gestational age at delivery, as well as infant sex, birth weight, appropriateness-for-gestational age (small, adequate, large), and 5-min Apgar score. The complete list of covariates along with their respective categorizations are presented in Supplementary Table 3.

\section{Statistical analysis}

Using descriptive statistics including frequencies and percentages, we first described the distribution of maternal and infant characteristics for each type of lethal congenital malformation. Then, the rates of inpatient medical diagnoses and clinical interventions were compared across malformation subtypes. Chi-square or Fisher's exact tests were used to identify statistically significant differences in the distribution of each characteristic or in the rate of each outcome between infants with a given birth defect vs. infants with none of the birth defects included in the study (the reference group). The Kaplan-Meier method was used to estimate 1-day, 7-day, 28-day, 90-day, 180-day, and 1-year survival with 95\% confidence intervals (CI), and to generate survival curves that were used to describe the risk of mortality during the first year of life by type of birth defect. Both the log-rank test and Wilcoxon test used to assess differences in survival across defect subtypes, the latter test placing more weight on differences in early survival.
To explore differences in hospital utilization by defect subgroup, we calculated the mean, per infant, number of inpatient days and costs of inpatient care, both during the birth hospitalization and during all inpatient encounters in the first year of life. To compare more extreme utilization, we also calculated the proportion of infants in each defect subgroup with $\geq 15$ days in the hospital or $\geq \$ 50,000$ in total costs. Statistically significant differences were compared using Wilcoxon rank-sum tests (continuous utilization measures) or chi-square and Fisher's exact tests (dichotomized utilization measures). All analyses were conducted using SAS 9.4 (SAS Institute, Inc., Cary, NC) using a 5\% type I error rate and two-sided hypothesis tests. Due to the rarity of the lethal malformations included in this study, only overall group frequencies and no counts fewer than 10 are presented in tables or text. The linked database was also de-identified prior to use and was approved by the Institutional Review Boards of the Florida Department of Health, University of South Florida, and Baylor College of Medicine.

\section{Results}

\section{Demographic characteristics}

Among 2,248,038 Florida-resident, live born infants, 786 had one of the birth defects of interest: T18 $(n=350,1.62 /$ 10,000 live births), T13 ( $n=206,0.97 / 10,000$ live births), anencephaly ( $n=125,0.56 / 10,000$ live births), bilateral renal agenesis $(n=53,0.24 / 10,000$ live births), skeletal dysplasias ( $n=38,0.17 / 10,000$ live births), and infants who were diagnosed with $>1$ of these birth defects $(n=14)$. The distribution of selected maternal and infant sociodemographic and clinical characteristics, overall and by type of birth defect, is presented in Supplementary Table 3. Mothers of infants with T13, T18, and multiple birth defects were more likely to be 35 years or older than those of noncase infants $(P<0.05)$, while mothers of infants with anencephaly were more likely to be teens $(22.4 \%$ vs. $11.0 \%$, $P<0.05)$. Mothers of infants with trisomies, bilateral renal agenesis, and skeletal dysplasias were more likely to be non-Hispanic black than mothers of unaffected infants, and foreign-born mothers tended to have higher trisomy and anencephaly rates than their US-born counterparts $(P<$ $0.05)$. Mothers of case infants had later entry to prenatal care, less education, and an increased likelihood of Cesarean delivery than mothers of non-cases. Gender distribution was similar within defect groups, with the exception of T18 (64.3\% females) and renal agenesis (58.5\% male). Gestational age and birth weight distributions of case infants were lower than non-case infants. 


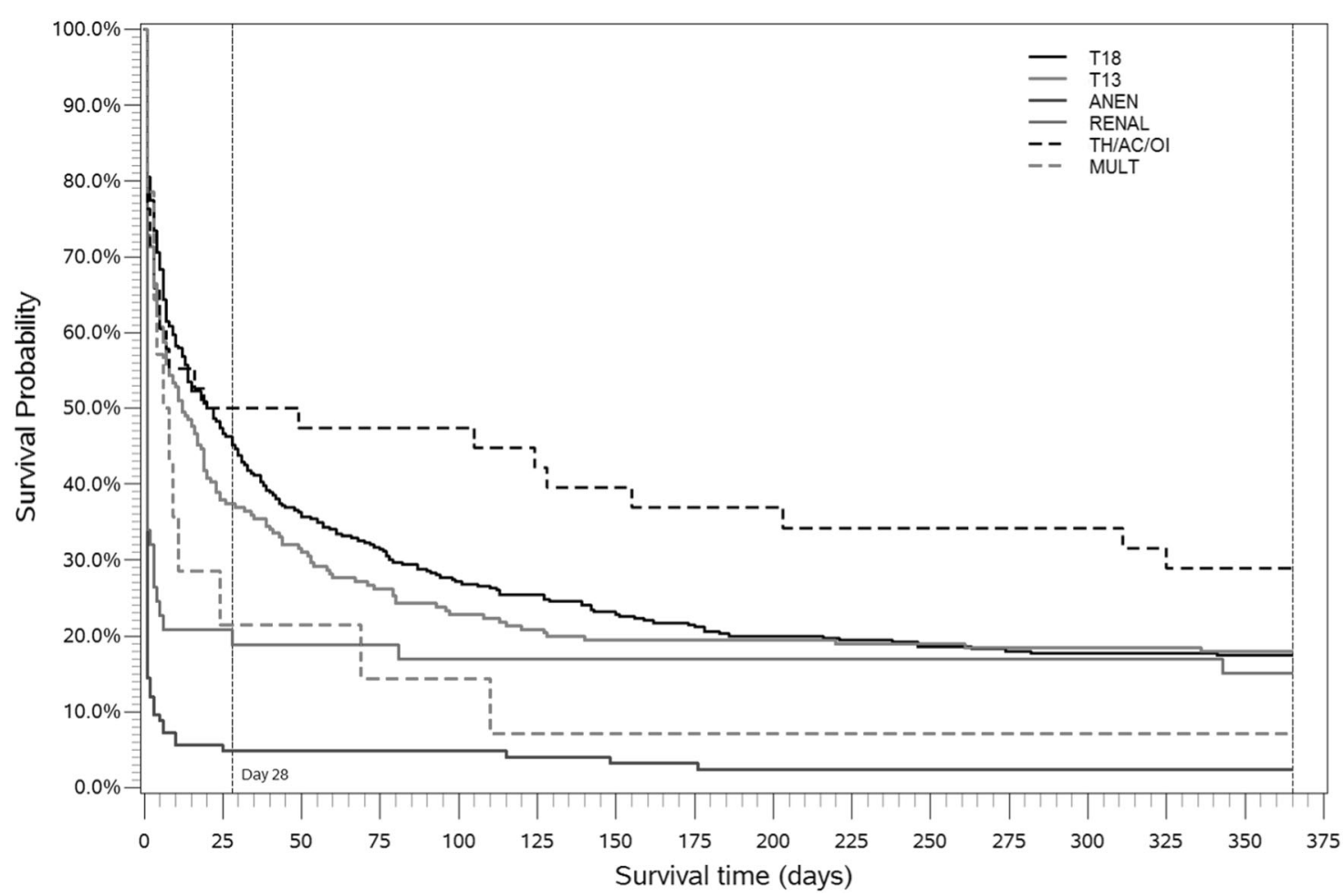

Fig. 1 Kaplan-Meier survival curves representing risk of mortality in the first year of life by type of birth defect, Florida, 1998-2009. This figure represents the survival curves that describe the risk of mortality during the first year of life by type of birth defect. The solid black curve represents trisomy 18 , the solid red curve represents trisomy 13 , the solid blue curve represents anencephaly, the solid green curve represents renal agenesis, the dotted black curve represents the skeletal dysplasias (thanatophoric dwarfism, achondrogenesis, and osteogenesis imperfecta), and the dotted red curve represents the $>1$ lethal

\section{Co-occurring morbidities and clinical interventions}

Congenital heart defects were the most frequent cooccurring birth defect in all birth defect groups except anencephaly. Only $1.4 \%$ of non-case infants were diagnosed with a congenital heart defect, compared to higher percentages in the multiple birth defects group $(71.4 \%, P<$ $0.05)$, T18 (63.7\%, $P<0.05)$, and T13 $(56.3 \%, P<0.05)$ (Supplementary Table 4). Infants with skeletal dysplasia had the highest rates of respiratory morbidities and respiratory interventions among all birth defect groups. All birth defect groups, except for infants with anencephaly, had statistically significantly higher rates of intubation (38.3-78.9\% of cases vs. $2.3 \%$ of non-cases, $P<0.05)$ and of invasive mechanical ventilation $(44.3-92.1 \%$ in cases vs. $2.1 \%$ of non-cases, $P<0.05$ ).

While infants in all birth defect groups had surgical interventions performed during their hospitalization, those with T13 (approximately 1-14\%), T18 (approximately 1$10 \%$ ) and skeletal dysplasia (approximately 3-34\%) underwent the highest number of surgical procedures. Across all birth defect groups, gastrointestinal surgeries were most malformation group (MULT). T18 trisomy 18, T13 trisomy 13, ANEN anencephaly, RENAL renal agenesis with oligohydramnios, TH/AC/ OI thanatophoric dwarfism, achondrogenesis, or osteogenesis imperfecta with agenesis, hypoplasia, or dysplasia of the lung, and indication of respiratory failure, MULT more than one of the birth defects included in the study. Note: Both the log-rank and Wilcoxon tests indicated statistically significant differences in one-year survival across defect types $(P<0.01)$

prevalent, with percutaneous gastrostomy tube insertions being the most common (ranging from 5.7-34.2\%). Infants with skeletal dysplasia had the highest percentage of tracheostomy placement $(23.7 \%)$. Infants with $\mathrm{T} 18$ or $\mathrm{T} 13$ were the only groups that underwent cardiac surgery but this occurred among a small percentage of the group (approximately $1-2 \%$ ). Among infants with $\mathrm{T} 13,31.1 \%$ were born with a cleft lip and palate defect; of those $3.4 \%$ underwent repair. Infants with anencephaly had the fewest non-surgical and surgical interventions among all groups.

\section{Survival and timing of death}

Figure 1 shows the Kaplan-Meier survival curves for each defect group with corresponding median survival and various time-specific survival estimates in Table 1. Infants with skeletal dysplasia had the highest 1-year survival rate (28.9\%), followed by T13, T18, and bilateral renal agenesis, which had similar 1-year survival rates $(18 \%, 17.4 \%$, and $15.1 \%$, respectively). Infants with anencephaly had the lowest survival rate at each time point evaluated. Infants with anencephaly or bilateral renal agenesis had the highest 

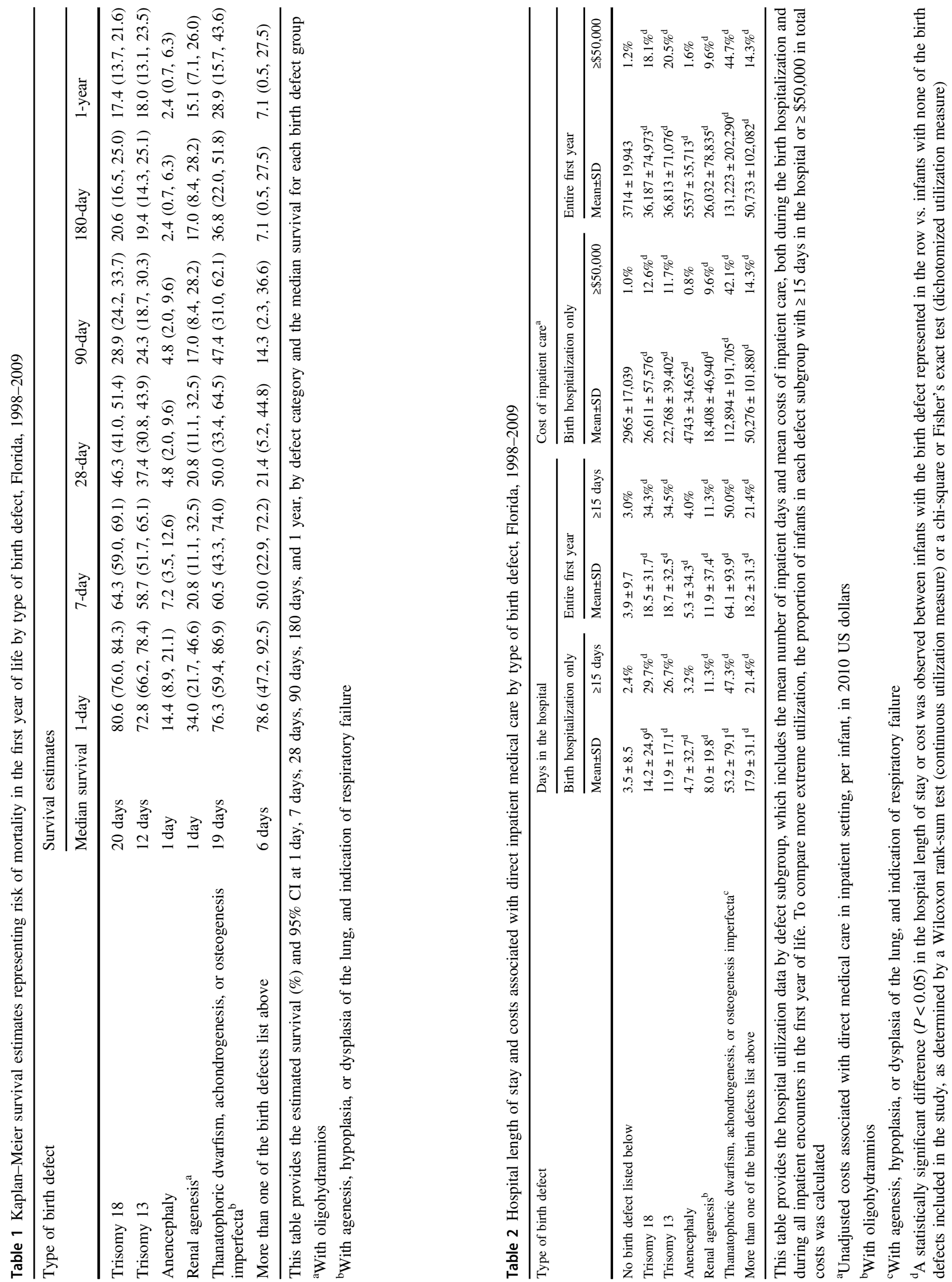


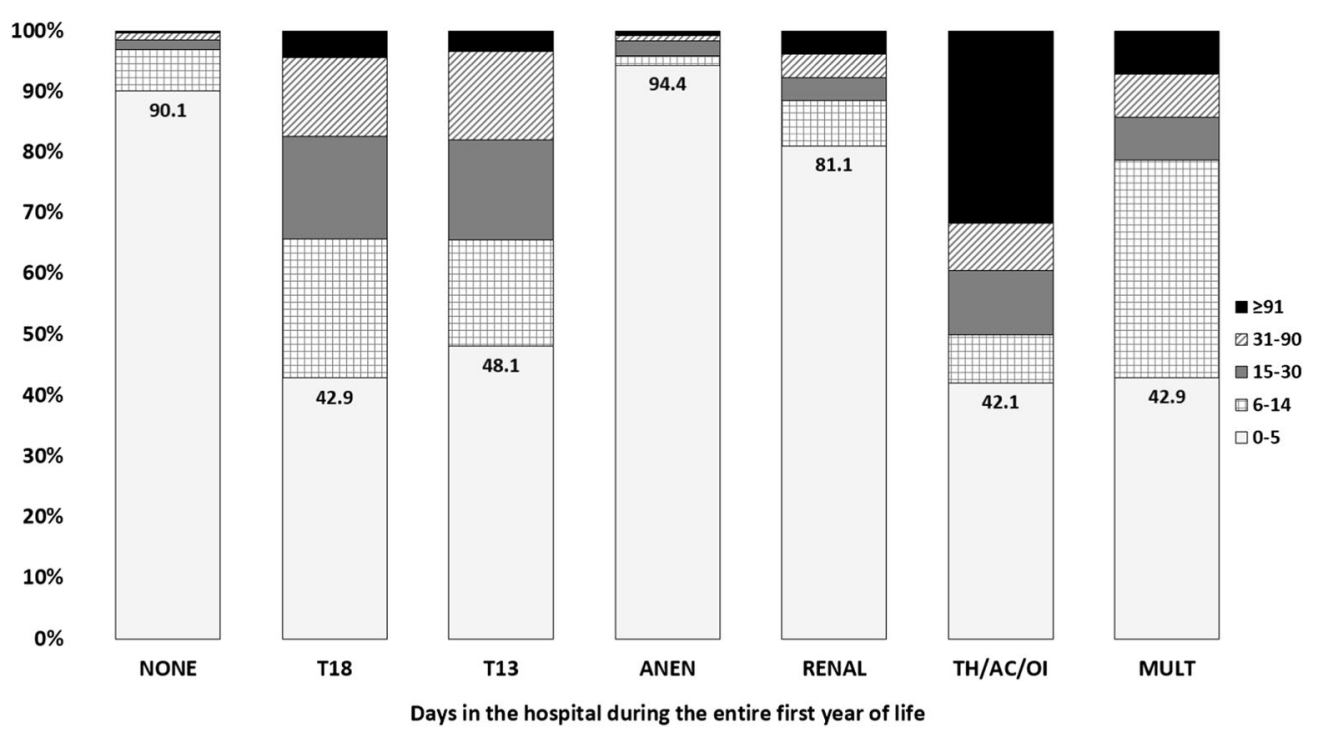

Fig. 2 Hospital length of stay associated with direct inpatient medical care by type of birth defect, Florida, 1998-2009. This figure represents the inpatient hospital length of stay, categorized from anywhere between 0 to greater than 90 days, for each birth defect group and noncase infants. The percentages displayed in each bar represent the proportion of all infants in that group who spent 5 or fewer days in receiving inpatient care during the first year of life. NONE none of the

mortality rate within the first day of life $(85.6 \%$ and $66 \%$, respectively) (Supplementary Table 3) and the lowest median age of survival (median survival 1 day) (Table 1).

\section{Length of hospitalization and discharge disposition}

With the exception of infants with anencephaly, those in all birth defect groups spent significantly more time in the hospital during their first year of life than non-case infants (mean LOS: 11.9-64.1 vs. 3.9 days for case vs. non-case infants respectively, $P<0.05$ ) (Table 2). Notably, $31.6 \%$ of infants with skeletal dysplasia spent more than 90 days in the hospital during their first year of life compared to $0.3 \%$ of non-case infants $(P<0.05)$. Over $80 \%$ of infants with anencephaly or bilateral renal agenesis had hospitalizations of 5 days or less (Fig. 2).

The majority of infants in all birth defect groups were discharged home except for those with anencephaly, who had fairly equal disposition to home or hospice (6.4\% and $5.6 \%$, respectively). Approximately 5-6\% of infants with $\mathrm{T} 18$, T13, or anencephaly received hospice care (Supplementary Table 3) and had palliative care encounters during their hospitalization (Supplementary Table 4).

\section{Costs of direct inpatient care}

Except for infants with anencephaly, those in all birth defect groups had significantly higher inpatient costs during the first year of life than non-case infants (mean hospital costs birth defects included in the study, T18 trisomy 18, T13 trisomy 13, ANEN anencephaly, RENAL renal agenesis with oligohydramnios, TH/AC/OI thanatophoric dwarfism, achondrogenesis, or osteogenesis imperfecta with agenesis, hypoplasia, or dysplasia of the lung, and indication of respiratory failure, MULT more than one of the birth defects included in the study

$\$ 26,032-\$ 131,223$ vs. $\$ 3714$ for case and non-case infants, respectively $P<0.05$ ) (Table 2 ). Hospital care costs during the first year of life exceeded $\$ 250,000$ for $21 \%, 7.1 \%$ and $5.7 \%$ of infants with skeletal dysplasia, multiple birth defects, and bilateral renal agenesis, respectively, vs. $0.1 \%$ of non-case infants $(P<0.05)$. In contrast, infants with anencephaly had the lowest total costs of hospitalization with $76 \%$ incurring less than $\$ 1000$ (Fig. 3). The mean cost of inpatient care for the first year of life for infants with anencephaly neared the mean cost of inpatient care for the first year of life for infants without birth defects (\$5537 vs. $\$ 3714$ respectively) (Table 2).

\section{Discussion}

In this large, population-based study we describe the inpatient hospital care, medical and surgical therapies, survival, and healthcare resource utilization in the first year of life for infants born in Florida with the congenital malformations most widely considered to be lethal: T18, T13, anencephaly, bilateral renal agenesis, thanatophoric dysplasia, achondrogenesis, and neonatal lethal osteogenesis imperfecta. Case infants received inpatient medical care, underwent various medical and surgical interventions, had higher survival rates compared to prior studies, and experienced increased healthcare costs and longer LOS compared to infants without birth defects; though, this generalization did not hold for infants born with anencephaly. Thus, using the 


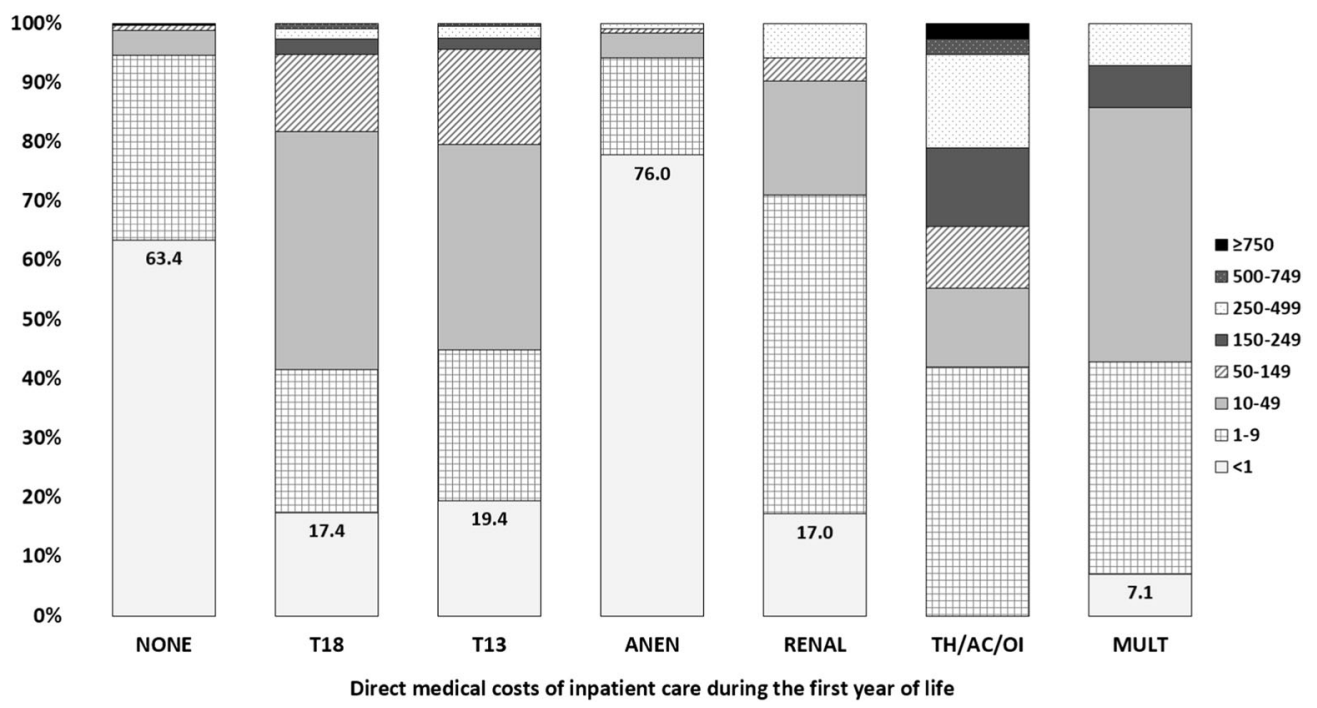

Fig. 3 Costs associated with direct inpatient medical care by type of birth defect, Florida, 1998-2009. This figure represents costs expressed as unadjusted costs associated with direct medical care in inpatient setting, per infant, in thousands of 2010 US dollars. Costs are categorized from anywhere between $<\$ 1000$ to $\geq \$ 750,000$, for each birth defect group and non-case infants. Percentages displayed in each bar represent the proportion of all infants in that group whose direct medical costs of inpatient care during the first year of life totaled less

term lethal may no longer be appropriate in describing some of these infants and could lead to miscommunication with families and inconsistent decision-making [2]. These results should be used in counseling families with fetuses or newborns affected with these diagnoses to reach a balanced, well-informed decision regarding the use of intervention, comfort care, perinatal hospice, or alternative forms of management.

\section{Survival and outcomes}

\section{Skeletal dysplasias}

Although the three most common skeletal dysplasias (thanatophoric dysplasia, achondrogenesis, and neonatal lethal osteogenesis imperfecta) comprise $40-60 \%$ of all lethal skeletal dysplasias, these diagnoses are rare. The published literature indicates that death occurs before or during the neonatal period, usually secondary to respiratory failure [22-25]. We observed higher survival rates, with 50\% survival to the neonatal period and $28.9 \%$ survival to 1 year of life.

The higher survival rates for infants with skeletal dysplasias in our cohort could be linked to the finding that these infants had more procedures than other birth defect groups in our study. These include intubation (78.9\%), mechanical ventilation $(92.1 \%)$, and tracheostomy placement $(23.7 \%)$. They also had the longest hospital LOS among all birth defect groups, incurred the highest hospital costs, had the than $\$ 1000$. The TH/AC/OI group did not have any infants with costs less than $\$ 1000$. NONE none of the birth defects included in the study, T18 trisomy 18, T13 trisomy 13, ANEN anencephaly, RENAL renal agenesis with oligohydramnios, TH/AC/OI thanatophoric dwarfism, achondrogenesis, or osteogenesis imperfecta with agenesis, hypoplasia, or dysplasia of the lung, and indication of respiratory failure, MULT more than one of the birth defects included in the study

highest $\mathrm{C}$-section rate, experienced the highest percentage of chronic lung disease, respiratory failure, pulmonary hypertension, sepsis, and pneumonia, and maintained the second highest seizure rates. Two case reports describe increased survival for three children with thanatophoric dysplasia, aged 4-9 years, who received life-sustaining equipment but had significant medical sequelae, including chronic ventilator dependence via tracheostomy, hydrocephalus with ventriculoperitoneal shunt placement, seizure disorders, profound developmental delay, and growth failure [26, 27]. Our data and the case report outcomes suggest that while aggressive treatment may increase life expectancy, there is a concomitant increase in morbidity and healthcare utilization.

\section{Bilateral renal agenesis}

Bilateral renal agenesis is considered incompatible with extrauterine life, with a median postnatal survival of $<24 \mathrm{~h}$; less than $5 \%$ of infants survive beyond 1 week [2]. Fetal losses are common, frequently due to cord compression, and newborns die within hours of birth due to severe respiratory insufficiency and severe pulmonary hypoplasia secondary to prolonged anhydramnios [28]. Despite a very poor prognosis, the 1 week survival rate of $20.8 \%$ in our study is considerably higher than reported in previous literature. However, the median survival of 1 day is similar to national rates [2]. While we were unable to find comparable 1-year survival rates in the literature, we observed a $15.1 \%$ 
1-year survival rate, which may be due to some infants receiving aggressive intervention: nearly $55 \%$ were intubated at birth and placed on invasive mechanical ventilation, $23 \%$ received chest tube placement, and $6 \%$ underwent peritoneal dialysis. The longest surviving child with bilateral renal agenesis is a 9-month-old infant described by Bienstock et al. [28]. In this case, the mother underwent serial amnioinfusions as a means of preventing pulmonary hypoplasia, the infant was born at 29 weeks with normal lung volumes, and underwent peritoneal dialysis at 36 hours of life. Even if pulmonary hypoplasia can be prevented by amnioinfusion, dialysis as a channel to renal transplantation is the only treatment that can ensure survival [28]. Peritoneal dialysis carries significant risks, with a very high mortality risk incurred when started before 3 months of age [28, 29].

\section{Anencephaly}

Anencephaly is one of the most fatal congenital malformations. The survival rates in our study are consistent with previous literature, with $85.6 \%$ of infants dying within the first $24 \mathrm{~h}$ of life. The 1 week and median survival rates (0-14\% and $\sim 1-24 \mathrm{~h}$, respectively) are also comparable with other studies [2, 30]. There are few case reports of anencephalic infants with longer survival, with the longest documented survival to 28 months of life [30, 31]. Given the extremely poor prognosis for these infants, it is not surprising that infants with anencephaly in our study had the fewest medical and surgical procedures, lowest 5 min Apgar score, and lowest survival. These findings support the American Academy of Pediatrics Neonatal Resuscitation Guidelines listing anencephaly as a condition for which non-initiation of resuscitation is appropriate [32].

\section{Trisomy 13 and 18}

T18 and T13 are the second and third most commonly diagnosed trisomies in live born patients after trisomy 21 . While in previous years medical treatments for trisomy 21 were withheld because of the associated congenital developmental delays and handicaps, the provision of intensive care for these infants has now been standard for decades [13]. However, there has been much controversy and debate on the management of infants with T13 or T18, resulting in numerous studies on survival and outcomes. Some studies have recommended that families not be offered intensive care due to the poor prognosis of these infants, with only about $50 \%$ of infants with T13 or T18 survived the first week of life, and approximately $5-10 \%$ survived through the first year of life [33-37]. More recent studies provide evidence of increased survival when significant medical and surgical inventions are provided [4, 6-14]. In comparison to these recent studies that have reported increased survival with intensive care, our study demonstrated similar survival rates for T18 or T13. Our 1-year survival rate for infants with T18 is analogous to that of these other studies $(17.4 \%$ vs.12-25\%, respectively), however our median survival rate is higher than other studies (20 days vs. 8-14 days, respectively) [2, 6, 8, 12]. Our 1-year survival rate and median survival for infants with T13 is comparable that of these other studies (18\% vs.12-37\%, respectively, and 12 days vs. 5-12 days, respectively) [2, 6, 12, 14]. Recent studies have also reported survival rates for 5 to 10 years of life [6, 12].

The perception of providers has also changed over time. McGraw et al. [33] found that a high proportion of US neonatal providers would consider resuscitating a 36-week infant prenatally diagnosed with T18 and a congenital heart defect, with maternal preference being the main reason to initiate resuscitation. Other studies that have surveyed parents of children with T18 or T13 and found that many parents observed that their children experience a higher quality of life and less pain and suffering than their care providers predicted [3, 4]. Although we were unable to assess intellectual disability in our cohort, others have reported that although individuals with T13 or T18 are generally severely or profoundly handicapped, these patients can continue to make modest developmental progress [3, 4, 9, 38]. However as providers, we should continue to have unbiased discussions with families on quality of life, taking into account their values and wishes but also continue to educate parents that despite aggressive interventions, improved quality of life is not a guarantee.

\section{Healthcare utilization}

Our study has evaluated the impact of lethal congenital malformations on the cost of providing direct inpatient medical care. Courtwright et al. [34] also estimated costs of direct medical care for infants with varying lethal congenital malformations and reported a range of $\$ 0-\$ 70,130$ for the cost of medical treatment from delivery until death or discharge, with a median cost of $\$ 4541$. We report mean costs of inpatient care ranging from \$5537-\$131,223 for only the first year of life for the entire cohort. While Courtwright et al. [34] provided median costs until death or discharge, our estimate for mean costs for the first year of life suggests that our costs are higher. Increased costs in our study could be related to longer survival, more intensive interventions, and thus longer hospital LOS. Another contributing factor could be the distribution of infants in the cohorts. Our study showed the highest costs of care were for infants with skeletal dysplasias. However, this group of infants represented just $2 \%$ of Courtwright's cohort. In addition, we found the lowest costs of care for infants with anencephaly, 
and in Courtwright's study, infants with lethal CNS malformations, which includes anencephaly, comprised the second largest group (24\%). In today's age of increasing hospital care costs, providers must consider the potential burden of these expenses on the community, as it is ethically and socially irresponsible to consume resources that do not provide inherent medical benefits to the patient.

\section{Hospice and palliative care}

In a survey of 653 neonatologists across the US and Canada, approximately 95\% endorsed the benefits of palliative care programs, although many reported barriers to supporting these programs including stigma, funding, difficulty with implementation, and lack of evidence of palliative care efficacy [39]. However, studies have shown that the impact of a palliative care consultation and the development and implementation of guidelines for the provision of palliative care in the NICU have enhanced end-of-life care [40-44]. Samsel et al. [44] found that implementation of a palliative care initiative that included provider education, written practice guidelines, and an electronic medical record order set led to an increase in re-direction to comfort care and increased palliative medication usage for infants in their final $48 \mathrm{~h}$ of life.

In addition, palliative care discussions have also included the consideration and organization of hospice care. Nelson et al. [7] found that infants with T18 who had disposition to home health, which includes hospice, nearly doubled over the study period. Among infants with selected birth defects in our study, only infants with T13, T18, or anencephaly received hospice care $(6.6 \%, 5.3 \%$, and $5.6 \%$ respectively). These findings clarify that the possibility of longer than anticipated survival has motivated the provider and family to make goal-oriented decisions regarding the care of their infant, including discussions of hospice care and extraordinary measures.

\section{Strengths and limitations}

There results should be interpreted after considering a few limitations. This study relies on administrative data, not collected for research purposes, linked to create a multi-year longitudinal database. The use of ICD-9-CM codes to identify infants with birth defects and outcomes is subject to human error, misdiagnosis, and improper identification due to the dependence on physician documentation in the medical record, coders' translation of information into appropriate ICD-9-CM codes, and correct documentation of those codes. Inherent problems with the lack of specificity of ICD-9-CM codes, particularly the codes for the skeletal dysplasias and bilateral renal agenesis, which include diagnoses that are considered benign conditions, also complicate these data. For these conditions, in order to ensure we were considering lethal diagnoses, a combination of diagnosis codes were used, rather than solely relying on the specific birth defect code. We also restricted the evaluation to 1 year of life in order to increase the sample size of each birth defect group by identification of the infants in each year over the entire 9 years of data analysis. Also, we did not have clinician review of records of all diagnoses of interest or access to review the specific details concerning severity of the malformations. Thus, extrapolating these data to larger populations beyond 1 year of life is fraught with potential bias. Another limitation of relying on the use and linkage of administrative data is the likelihood of not capturing birth hospitalizations among infants with lethal defects who died immediately after birth or died before the first day of life. These infants are less likely to generate a hospital discharge record to be linked, and therefore to not be classified as a case. Furthermore, this study did not include cases diagnosed prenatally that may have been electively terminated or spontaneously aborted, resulting in a higher number of live born infants with congenital malformations with less severe phenotypes. This, along with the inability to disproportionately capture deaths occurring immediately after birth, may have resulted in overestimated survival rates. Our observed survival rates for infants with T13 or T18 may be overestimated as well, given the inability of the coding system to distinguish between full trisomy and partial or mosaic variants; infants with partial or mosaic trisomies have longer survival than those with full trisomy [6]. Our study was also limited due to lack of availability of outcome data after 2010. Future research should assess the extent to which our findings may have changed in recent years. Lastly, we were not able to investigate whether treatment intensity may have impacted survival among infants with lethal conditions. Two recent studies have evaluated treatment intensity and survival in infants with lethal congenital malformations and both concluded that aggressive obstetric or neonatal care did not prolong survival $[34,36]$. These studies continue to raise the question of how much care is "the right amount", given the poor prognosis of these children [10].

This study has several strengths. Birth defect cases were identified from a large, ethnically diverse, longitudinally linked statewide database that includes data on over 2 million live births during a 12-year period. The large sample size allowed for the assessment of survival and healthcare utilization practices for several birth defects in which previous findings were based largely on case series reports (e.g., bilateral renal agenesis, anencephaly, thanatophoric dysplasia, achondrogenesis and neonatal lethal osteogenesis imperfect). These studies often include a limited amount of information about the infants' medical courses and practices, which vary broadly across different countries and 
sites. Additionally, the large sample size, compared to previous studies of live born infants, also enabled us to investigate several maternal and infant risk factors potentially related to survival that had not been previously studied.

\section{Implications and conclusion}

The results of this study have significant implications for the care and management of infants considered to have lethal malformations. The evolution of these lethal birth defects and their medical management has led to a shift to a new era of the consideration of providing life-prolonging medical interventions, especially for infants with T13 or $\mathrm{T} 18$, raising significant ethical and financial concerns. The current practice of some providers is to withhold lifesustaining treatment and counsel parents based on historical precedence without considering current data regarding natural progression and survival. Impartial discussions regarding quality of life are rarely held. We support the shift in the paradigm to a model of shared decision-making that actively engages the expertize and perspectives of parents and providers, incorporating current medical literature with the values of the family to assist in shaping goals of care at critical time points prenatally, perinatally, and postnatally [45]. Providers who counsel families of these infants should continue to stress that these diagnoses are associated with poor prognoses; however, all potential outcomes should be discussed, including variable survival. Parents should be counseled to be prepared to care for a critically ill infant if they choose to continue the pregnancy, understanding that multiple invasive procedures can be painful, psychologically traumatic, and do not guarantee long-term normal outcomes or improved quality of life. Regarding longer than anticipated survival, the provider and family again should make goal-oriented decisions on how to care for the infant, including discussions of involvement of hospice and palliative care as well as the possible provision of extraordinary measures [30]. We also support a palliative and hospice approach, if consistent with families' wishes, for infants with very poor prognoses, given the potential of the infant experiencing pain and suffering without significant medical benefit and allocation of scarce resources [2, 45]. Hospice and Palliative Care medicine teams should be considered during these discussions as they can assist the providers with these often difficult conversations. These teams can be of most benefit when a prenatal diagnosis is discovered.

This study evaluated healthcare utilization by hospital LOS and healthcare costs of specific groups of infants, therefore, more comparative studies are needed. Studies evaluating the home medical care system for these infants, examining intensity of intervention, and costs after hospital discharge as well as the benefits of hospice care are also needed, as are studies evaluating both quality of life in infancy and childhood for infants with life-limiting diagnoses.

In summary, this population-based study describes outcomes, survival rates, and healthcare utilization of patients with specific congenital malformations considered lethal, using a linked database in Florida. We conclude that infants with T18, T13, bilateral renal agenesis, thanatophoric dysplasia, achondrogenesis or neonatal lethal osteogenesis imperfecta who undergo medical and surgical interventions, have increased survival rates compared with infants described in previous population studies and case reports. However, they have the potential for longer hospital stays and increased hospital care costs, especially in the case of infants with skeletal dysplasias. We hope that this information will help providers and parents make more informed decisions related to the management of the pregnancy, fetus and neonate, keeping in mind the varying survival outcomes, appropriate use of healthcare resources, and consideration of the best interest of the infant. Also we hope that this study will assist with the formation of best practice recommendations for the inpatient hospital care of these infants, and will impact healthcare policy and practice for this population.

\section{Compliance with ethical standards}

Conflict of interest The authors declare that they have no conflict of interest.

\section{References}

1. Wilkinson DJ, Thiele P, Watkins A, De Crespigny L. Fatally flawed? A review and ethical analysis of lethal congenital malformations. BJOG. 2012;119:1302-8.

2. Wilkinson D, de Crespigny L, Xafis V. Ethical language and decision-making for prenatally diagnosed lethal malformations. Semin Fetal Neonatal Med. 2014;19:306-11.

3. Janvier A, Farlow B, Wilfond BS. The experience of families with children with trisomy 13 and 18 in social networks. Pediatrics. 2012;130:293-8.

4. Kosho T, Kuniba H, Tanikawa Y, Hashimoto Y, Sakurai H. Natural history and parental experience of children with trisomy 18 based on a questionnaire given to a Japanese trisomy 18 parental support group. Am J Med Genet A. 2013;161A:1531-42.

5. Baty BJ, Jorde LB, Blackburn BL, Carey JC. Natural history of trisomy 18 and trisomy 13: II. Psychomotor development. Am J Med Genet. 1994;49:189-94.

6. Meyer RE, Liu G, Gilboa SM, Ethen MK, Aylsworth AS, Powell CM, et al. Survival of children with trisomy 13 and trisomy 18: A multi-state population-based study. Am J Med Genet A. 2016;170A:825-37.

7. Nelson KE, Hexem KR, Feudtner C. Inpatient hospital care of children with trisomy 13 and trisomy 18 in the United States. Pediatrics. 2012;129:869-76.

8. Kosho $T$, Nakamura $T$, Kawame $H$, Baba A, Tamura M, Fukushima Y. Neonatal management of trisomy 18: clinical 
details of 24 patients receiving intensive treatment. Am J Med Genet A. 2006;140:937-44.

9. Ishitsuka K, Matsui H, Michihata N, Fushimi K, Nakamura T, Yasunaga $\mathrm{H}$. Medical procedures and outcomes of Japanese patients with trisomy 18 or trisomy 13: analysis of a nationwide administrative database of hospitalized patients. Am J Med Genet A. 2015;167A:1816-21.

10. Carey JC. Perspectives on the care and management of infants with trisomy 18 and trisomy 13: striving for balance. Curr Opin Pediatr. 2012;24:672-8.

11. Carey JC, Kosho T. Perspectives on the care and advances in the management of children with trisomy 13 and 18. Am J Med Genet C Semin Med Genet. 2016;172:249-50.

12. Nelson KE, Rosella LC, Mahant S, Guttmann A. Survival and surgical interventions for children with trisomy 13 and 18. JAMA. 2016;316:420-8.

13. McCaffrey MJ. Trisomy 13 and 18: Selecting the road previously not taken. Am J Med Genet C Semin Med Genet. 2016;172:251-6.

14. Tsukada K, Imataka G, Suzumura H, Arisaka O. Better prognosis in newborns with trisomy 13 who received intensive treatments: a retrospective study of 16 patients. Cell Biochem Biophys. 2012;63:191-8.

15. Pine M, Kowlessar NM, Salemi JL, Miyamura J, Zingmond DS, Katz NE, et al. Enhancing clinical content and race/ethnicity data in statewide hospital administrative databases: Obstacles encountered, strategies adopted, and lessons learned. Health Serv Res. 2015;50:1300-21.

16. Salemi JL, Salinas-Miranda AA, Wilson RE, Salihu HM. Transformative use of an improved all-payer hospital discharge data infrastructure for community-based participatory research: A sustainability pathway. Health Serv Res. 2015;50:1322-38.

17. Salemi JL, Tanner JP, Bailey M, Mbah AK, Salihu HM. Creation and evaluation of a multi-layered maternal and child health database for comparative effectiveness research. J Regist Manag. 2013;40:14-28.

18. Alexander GR, Himes JH, Kaufman RB, Mor J, Kogan M. A United States national reference for fetal growth. Obstet Gynecol. 1996;87:163-8.

19. Coffey RMBM, Steiner S. Final report observation status related to hospital records. HCUP Methods Series Report \#2002-3. Agency for Healthcare Research and Quality, Rockville, MD; 2002.

20. Salemi JL, Comins MM, Chandler K, Mogos MF, Salihu HM. A practical approach for calculating reliable cost estimates from observational data: application to cost analyses in maternal and child health. Appl Health Econ Health Policy. 2013;11: 343-57.

21. US Department of Labor: Bureau of Labor Statistics. Consumer Price Index: all urban consumers-(CPI-U). 2010. Available from: http://www.bls.gov/data.

22. Camera G, Mastroiacovo P. Birth prevalence of skeletal dysplasias in the Italian Multicentric Monitoring System for Birth Defects. Prog Clin Biol Res. 1982;104:441-9.

23. Orioli IM, Castilla EE, Barbosa-Neto JG. The birth prevalence rates for the skeletal dysplasias. J Med Genet. 1986;23: 328-32.

24. Ayadi ID, Hamida EB, Rebeh RB, Chaouachi S, Marrakchi Z. Perinatal lethal type II osteogenesis imperfecta: a case report. Pan Afr Med J. 2015;21:11.

25. Donnelly DE, McConnell V, Paterson A, Morrison PJ. The prevalence of thanatophoric dysplasia and lethal osteogenesis imperfecta type II in Northern Ireland - a complete population study. Ulst Med J. 2010;79:114-8.
26. Baker KM, Olson DS, Harding CO, Pauli RM. Long-term survival in typical thanatophoric dysplasia type 1 . Am J Med Genet. 1997;70:427-36.

27. MacDonald IM, Hunter AG, MacLeod PM, MacMurray SB. Growth and development in thanatophoric dysplasia. Am J Med Genet. 1989;33:508-12.

28. Bienstock JL, Birsner ML, Coleman F, Hueppchen NA. Successful in utero intervention for bilateral renal agenesis. Obstet Gynecol. 2014;124:413-5.

29. Alexander RT, Foster BJ, Tonelli MA, Soo A, Nettel-Aguirre A, Hemmelgarn BR, et al. Survival and transplantation outcomes of children less than 2 years of age with end-stage renal disease. Pediatr Nephrol. 2012;27:1975-83.

30. Dickman H, Fletke K, Redfern RE. Prolonged unassisted survival in an infant with anencephaly. BMJ Case Rep. 2016:1-3.

31. McAbee G, Sherman J, Canas JA, Boxer H. Prolonged survival of two anencephalic infants. Am J Perinatol. 1993;10:175-7.

32. American Heart Association. NRP: Textbook of Neonatal Resuscitation 6th edition: Lesson 9 Ethics and Care at the End of Life. American Academy of Pediatrics, 2011;9:287-8.

33. McGraw MP, Perlman JM. Attitudes of neonatologists toward delivery room management of confirmed trisomy 18: potential factors influencing a changing dynamic. Pediatrics. 2008;121:1106-10.

34. Courtwright AM, Laughon MM, Doron MW. Length of life and treatment intensity in infants diagnosed prenatally or postnatally with congenital anomalies considered to be lethal. J Perinatol. 2011;31:387-91.

35. Boghossian NS, Hansen NI, Bell EF, Stoll BJ, Murray JC, Carey JC, et al. Mortality and morbidity of VLBW infants with trisomy 13 or trisomy 18. Pediatrics. 2014;133:226-35.

36. Subramaniam A, Jacobs AP, Tang Y, Neely C, Philips JB 3rd, Biggio JR, et al. Trisomy 18: A single-center evaluation of management trends and experience with aggressive obstetric or neonatal intervention. Am J Med Genet A. 2016;170A:838-46.

37. Rasmussen SA, Wong LY, Yang Q, May KM, Friedman JM. Population-based analyses of mortality in trisomy 13 and trisomy 18. Pediatrics. 2003;111:777-84.

38. Bruns DA. Developmental status of 22 children with trisomy 18 and eight children with trisomy 13: implications and recommendations. Am J Med Genet A. 2015;167A:1807-15.

39. Cortezzo DE, Sanders MR, Brownell E, Moss K. Neonatologists' perspectives of palliative and end-of-life care in neonatal intensive care units. J Perinatol. 2013;33:731-5.

40. Pierucci RL, Kirby RS, Leuthner SR. End-of-life care for neonates and infants: the experience and effects of a palliative care consultation service. Pediatrics. 2001;108:653-60.

41. Leuthner SR, Pierucci R. Experience with neonatal palliative care consultation at the Medical College of Wisconsin-Children's Hospital of Wisconsin. J Palliat Med. 2001;4:39-47.

42. Carter BS, Bhatia J. Comfort/palliative care guidelines for neonatal practice: development and implementation in an academic medical center. J Perinatol. 2001;21:279-83.

43. Catlin A, Carter BS. Creation of a neonatal end-of-life palliativecare protocol. J Clin Ethics. 2001;12:316-8.

44. Samsel C, Lechner BE. End-of-life care in a regional level IV neonatal intensive care unit after implementation of a palliative care initiative. J Perinatol. 2015;35:223-8.

45. Andrews SE, Downey AG, Showalter DS, Fitzgerald H, Showalter VP, Carey JC, et al. Shared decision making and the pathways approach in the prenatal and postnatal management of the trisomy 13 and trisomy 18 syndromes. Am J Med Genet C Semin Med Genet. 2016;172:257-63. 